\title{
Viscoelastic Flow into a Slit at Low Reynolds Numbers
}

\author{
Part 1: Flow Patterns
}

\author{
By Kiyoji Nakamura, Shizuo Umegaki and Akira Horikawa, Member, TMSJ \\ Department of Mechanical Engineering, Osaka University, Suita, Osaka
}

Based on the Journal of the Textile Machinery Society of Japan, Transaction, Vol. 29, No. 5, T63-69 (1976)

\begin{abstract}
It is necessary to consider both relaxation time and viscosity for investigating viscoelastic flow. Polyethylene flow from a reservoir into a slit at low Reynolds numbers has been visually observed to investigate the flow patterns.

The results of this study are as follows:

When Reynolds number is smaller than 1, circulating secondary flow appears or disappears depending upon the conditions of the main flow and of the characteristics of melted polymers. The liquid having longer rexation time has a lower critical deformation velocity at which the circulating secondary flow occurs in the reservoir. The region of circulating secondary flow spreads out as the deformation velocity becomes higher. Although the slit entry angle changes, the patterns of main flow into the slit hardly change. In regard to the liquid having shorter relaxation time, irregular and unstable flow occurs at the inlet region of the slit before the circulating secondary flow appears.
\end{abstract}

\section{Introduction}

To understand the behavior of viscoelastic polymer melts is very important for processing them successfully in industry and for analysing the viscoelastic fluid dynamics. Polymeric flow from a reservoir into a capillary is different from that of Newtonian liquids. In 1956, Tordella ${ }^{[1]}$ found this difference by the phenomena that secondary circulating regions are observed near the entrance of a capillary into which a polymeric melt (low-density polyethylene) flows. Experimental results of melted polymers or polymer solutions other than low-density polyethylene have since been reported. Giesekus ${ }^{[2]}$ and Schümmer ${ }^{[3]}$ studied the flow patterns of dilute aqueous solutions of polyacrylamid and polyisobuthylene at the entrance of capillaries. Ballenger and White ${ }^{[4]}$ experimented similarly using polystylene, polyethylene and polypropylene. Bagley and Birks $^{[5]}$ observed no secondary circulating flow of a highdensity polyethylene melt at the entrance of capillaries. Experiments indicate that the flow patterns differ with polymeric materials. However, the influence of deformation velocity and temperature on flow patterns has not been studied to date. Ballenger and White ${ }^{[4]}$ reported, using low-density polyethylene at $180^{\circ} \mathrm{C}$, that the region of secondary flow increases with increasing the deformation velocity.

The studies above-mentioned are of axisymmetrical entrance flow into a capillary. On the other hand, $\mathrm{Han}^{[6,7]}$ recently studied a two-dimensional entrance flow into a slit, and concluded that polymeric melts exhibit no second- ary motions at low Reynolds numbers (using polyethylene, polypropylene and polystyrene).

Kaloni ${ }^{[10,11]}$ studied theoretically a two-dimensional converging flow into a slit and an axisymmetrical converging flow into a capillary by perturbation technique, and found that, in the latter, the effect of viscoelasticity depends upon the first order perturbation term, and in the former, upon the second order perturbation term. From these results, it may be said that the circulating secondary flow would be found less frequently with two-dimensional flow than with axisymmetrical flow.

The purpose of this paper is to present, at low Reynolds numbers, some experimental results of two-dimensional flow of polymeric melts in the entrance region of slits by changing deformation velocity, temperature, melt index of low-density polyethylene or wall angle.

\section{Characteristics of viscoelastic flow}

Viscoelastic fluids, different from Newtonian liquids, are so complicated in their flow behavior that many equations are suggested to analyze it. In general, the condition for establishing the law of dynamical similarity for a viscoelastic flow depends on the constitutive equations used.

Now, we consider a steady viscoelastic flow on the basis of a three-dimensional Maxwell model to explain the characteristics of a viscoelastic flow. In this case, viscoelastic flow fields are dynamically similar if Reynolds numbers, Weissenberg numbers and the geometrical boundaries are the same. Reynolds number $R \boldsymbol{e}$ and Weissenberg number 
$W e$ are defined in the form:

$$
\begin{aligned}
& R e=\frac{\rho V H}{\eta} . \\
& W_{e}=\frac{\lambda V}{H} \quad \cdots
\end{aligned}
$$

where $V$ is the representative velocity of the flow, $H$ the representative length, $\rho$ the density, $\eta$ the coefficient of viscosity and $\lambda$ the relaxation time.

In the present paper, the flow being limited within law Reynolds numbers (see Table 4), we use as secondary flow parameters the deformation velocity, the melt index and the temperature in connection with the Weissenberg number.

\section{Apparent viscosity and its measurement}

Melted polymers exhibit non-Newtonian viscosity. In this paper, non-Newtonian viscosity is measured by the capillary tube instrument. The power law model which is often used for melted polymers may be used to the relation between the rate of strain and the viscosity.

To obtain flow index $n$, the pressure loss per unit length $\Delta P / L$ and the flow rate $Q$ must be measured in the fully developed region. As the stress on the wall is in the form

$$
\tau_{w}=\frac{R}{2} \frac{\Delta P}{L}
$$

the rate of strain on the wall $\gamma_{w}$ and flow index $n$ are calculated by

$$
\begin{aligned}
& \dot{r}_{w}=\frac{4 Q}{\pi R^{3}}\left(\frac{3 n+1}{4 n}\right) \\
& \frac{1}{n}=\frac{d \log \left(\frac{4 Q}{\pi R^{3}}\right)}{d \log \tau_{w}}
\end{aligned}
$$

where $R$ is the tube radius. Flow indices thus obtained are shown in Table 1. Properties of polyethylene used in this experiment are shown in Table 2.

The apparent viscosity along the surface of parallel plates is

$$
\eta_{w}=\frac{\tau_{w}}{r_{w^{\prime}}}
$$

where

$$
\dot{r}_{w}=\frac{2 n+1}{3 n} \frac{6 Q^{\prime}}{H^{2}}
$$

and $H$ is the distance between parallel plates, $Q^{\prime}$ the flow rate per unit width of parallel plates.

From the apparent viscosity along parallel plates $\eta^{\prime} w$, Reynolds number is defined as

$$
R e=\frac{\rho \bar{V} H}{\eta_{w^{\prime}}^{\prime}}
$$

where $\bar{V}$ is the mean velocity between parallel plates.

\section{Relaxation time of melted polymers}

It is a fundamental characteristic of polymers that they have huge molecular weights $100-10000$ times larger than ordinary molecules. Thus, there is a correlation between the molecular weights and properties of polymers.

We can consider the relaxation time as an index of viscoelastic properties of polymers. Based on the molecular theory, there is proposed a relation between the relaxation time and the molecular weight of polymers in the form

$$
\lambda \sim \frac{\eta_{0} M}{\rho T}
$$

where $\lambda$ is the relaxation time, $\eta_{0}$ the limit viscosity in zero shear rate, $M$ the molecular weight, $\rho$ the density and $T$ the absolute temperature ${ }^{[8]}$. The higher the molecular weight is or the lower the temperature is, the larger the relaxation time becomes.

Thus, the molecular weight is a fundamental characteristic of polymers. But as it is difficult to measure the molecular weight, one uses generally as an index of molecular weight the melt index which can be measured easily. Between the molecular weight and the melt index, for example, there is a relation ${ }^{[9]}$

$\log ($ melt index $)=5.09-1.53 \times 10^{-4} \times($ mean molecular weight).

Table 1 Flow index $n$

\begin{tabular}{lllllllllll}
\hline Melt index & \multicolumn{2}{c}{0.5} & \multicolumn{4}{c}{4} & \multicolumn{2}{c}{20} & \multicolumn{2}{c}{35} \\
\hline Temperature $\left({ }^{\circ} \mathrm{C}\right)$ & 190 & 230 & 260 & 190 & 230 & 150 & 190 & 150 & 190 \\
\hline Flow index $n$ & 0.46 & 0.54 & 0.57 & 0.65 & 0.71 & 0.61 & 0.78 & 0.77 & 0.94 \\
\hline
\end{tabular}

Table 2 Properties of polyethylene

\begin{tabular}{lllllll}
\hline Melt & Melting & \multicolumn{3}{c}{ Density $\left(\mathrm{g} / \mathrm{cm}^{3}\right)$} \\
\cline { 4 - 6 } index & point $\left({ }^{\circ} \mathrm{C}\right)$ & $150^{\circ} \mathrm{C}$ & $190^{\circ} \mathrm{C}$ & $230^{\circ} \mathrm{C}$ & $260^{\circ} \mathrm{C}$ \\
\hline 0.5 & 114 & & & & \\
4 & 116 & & & & \\
20 & 118 & 0.784 & 0.762 & 0.742 & 0.727 \\
35 & 122 & & & & \\
\hline
\end{tabular}


The melted polyethylene with a small melt index has a high molecular weight.

From the fact described above, the relation between the melt index and the relaxation time is that a melted polymer with a small melt index has a long relaxation time, namely a highly viscoelastic property. Hence, in this paper, the melt index is used as an index of viscoelastic properties.

\section{Experimental apparatus and procedure}

The main components of the experimental apparatus are shown in Fig. 1, in which (1) is an electric motor, (2) reduction gears, (3) and (6) a worm and a worm wheel, (5) screws, (6) a plunger, (7) an extruder and (8) a converging die from which the melted polyethylene is extruded. The extruding speed is controlled from 0.742 to $248.8 \mathrm{~mm} / \mathrm{min}$ by changing reduction gears and the revolution of the electric motor.

Details of the extruder is shown in Fig. 2, in which (1) is extruder body, (2) a plunger, (3) a piston, (4) a die, (5) gaskets and (6) heat-resistant glass-plates. Flow patterns are observed in the region surrounded by broken lines. The reservoir width $H$ is $10.2 \mathrm{~mm}$, and the length to width ratio $W / H$ is 8.06 . In order to observe the polymer flow, heat-resistant glass windows are placed on both sides of the slit die.

All the metal surfaces of the reservoir and the slit die are covered with band electric heaters. The heating systems are separated into five sections, each controlled by means of a temperature controller within $\pm 0.5^{\circ} \mathrm{C}$. In order to minimize heat losses to surroundings, thick layers of asbestos are placed all over the heat surfaces. Temperature is measured by thermocouples.

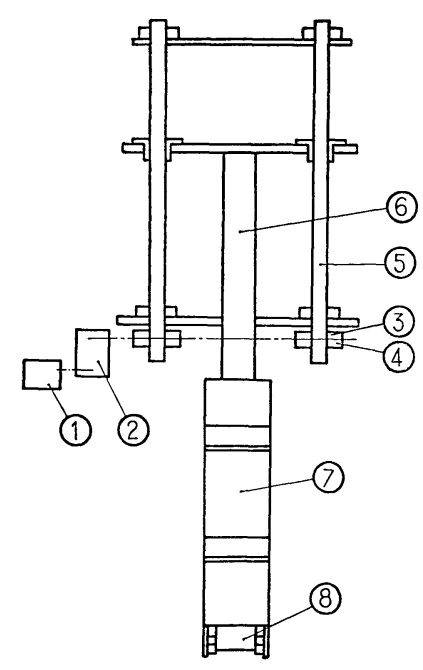

Fig. 1 Schematic diagram of the apparatus

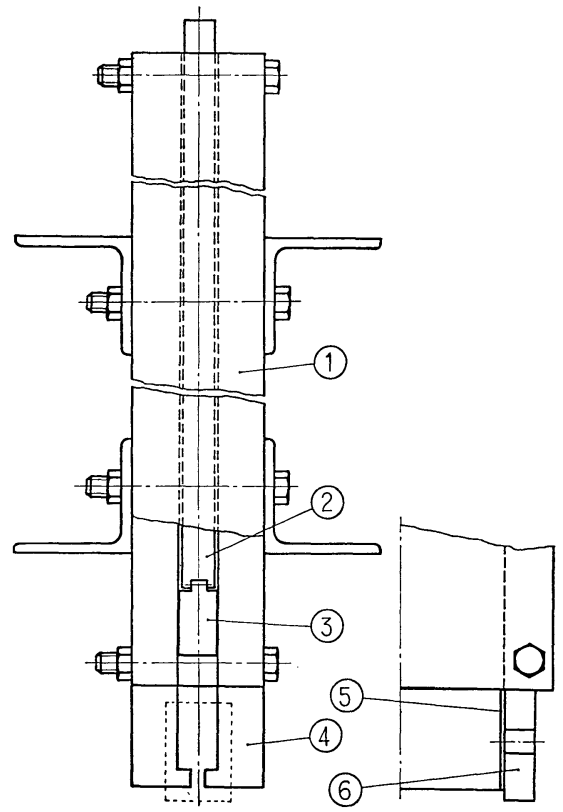

Fig. 2 Extruder

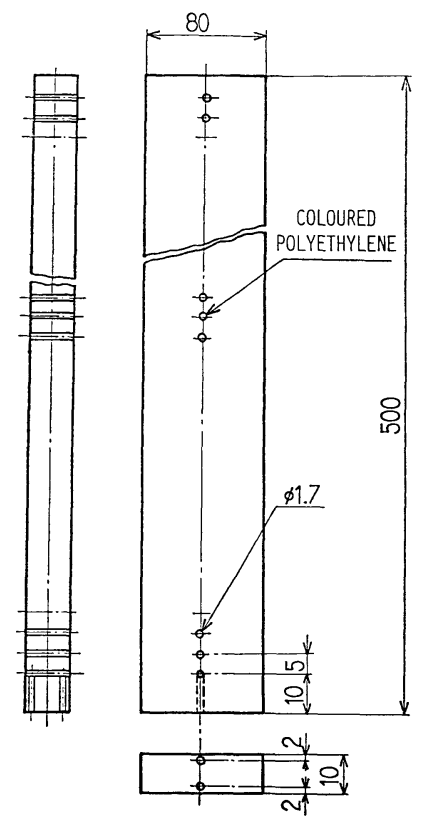

Fig. 3 Test sample of polyetylene

Vol. 23 No. 4 (1977) 
Polymer samples studied were premolded into $82 \mathrm{~mm} \times$ $10 \mathrm{~mm} \times 500 \mathrm{~mm}$ so that they would slide easily into the reservoir. Black filaments of the same polymer, obtained by adding carbon black and extruded from a $1 \mathrm{~mm}$ diameter hole were threaded through holes drilled at the interval of $5 \mathrm{~mm}$ on the center line of the polymer sample.

Experiments were carried out as follows: A polyethylene sample with markers on the center line was placed in the reservoir and heated up to a fixed temperature. After an equilibrium temperature was established, the pluger was forced down at a constant speed by means of the electric motor.

In order to assure the flow pattern to be two-dimensional, two reservoirs of $W / H=3.5$ and 8.06 were tried. As the same flow patterns were observed, it may be said that these flows were two-dimensional. So, only the reservoir of $W / H=8.06$ was used in this study. Materials used for the experiment were low-density polyethylene (MitsubishiYuka Co., HE-30, YK-50, MS-70 and MV-30).

\section{Flow patterns in flat entry}

We have observed flow patterns in a reservoir having a converging angle $\phi=90^{\circ}$ (shown in Fig. 4), changing deformation velocity, temperature and melt index. The flow patterns may be divided, according to the experimental conditions, into two broad classes shown in Figs. 5 and 6; flow patterns with and without circulating secondary motions. It should be noted here that, as Reynolds numbers in this experiment is much less than 1 , the flow pattern of a Newtonian liquid has no circulating secondary flow such as a wine glass.

In Table 3 is shown the relation between the existence of circulating secondary flow and deformation velocity $(\mathrm{V} / H)$.
Reynolds numbers in this experiment are shown in Table 4. Flow patterns with circulating secondary flow are shown by the sign $\bigcirc$, and that with no circulating secondary flow by the sign $\times$. From this table, circulating secondary flow is recognized only for melt index 0.5 , and is not for melt index 4, 20 and 35 within the limits of this experiment. Deformation velocities and Reynolds numbers for melt index 0.5 in these tables indicate either the lower limits for existing circulating secondary flow or the upper limits for disappearing circulating secondary flow. For melt indexes 4, 20 and 35, within the limits of deformation velocities in Table 3 or Reynolds numbers in Table 4, there is no circulating secondary flow of polymeric melts.

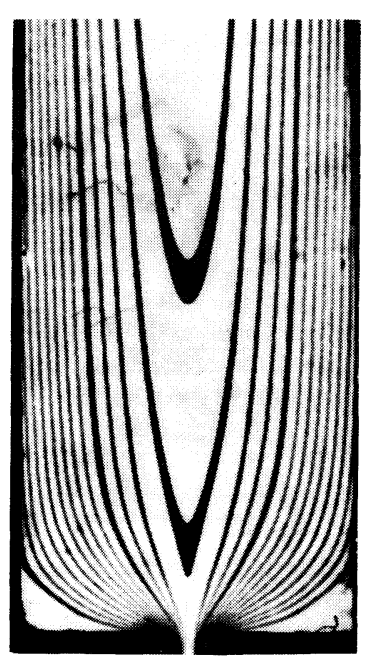

Fig. 5 Flow pattern with no-circulating secondary flow

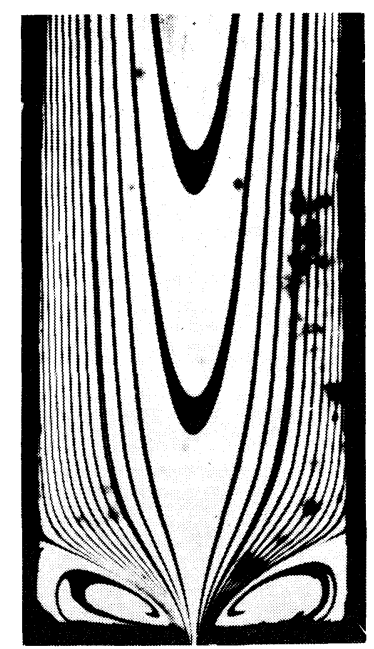

Fig. 4 Flow pattern near a slit

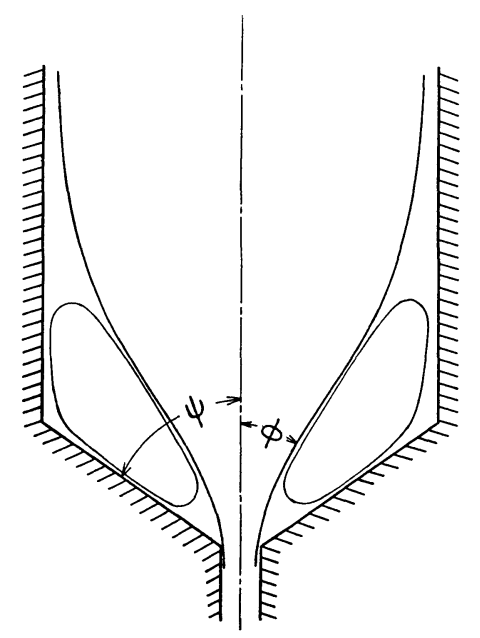


From these results, it may be said that, even at the same deformation velocity, there is circulating secondary flow for small melt index (substance of large relaxation time), but none for large melt index. Polymers in this case flow almost radially inward into the slit such as viscous fluids. Thus, the critical deformation velocity at which the secondary flow appears becomes larger with increasing the melt index (decreasing the relaxation time). We will further study this point by changing temperature.

6.1 For melt index 0.5

a. Influence of deformation velocity

The stream line with no circulating secondary flow is convex to the bottom as shown in Fig. 5, but that with circulating secondary flow is concave to the bottom such as shown in Fig. 6 by pushed up by secondary flow. This flow pattern is similar to axisymmetrical flow patterns obtained by Bagley et al. ${ }^{5)}$ In Fig. 7 is shown the flow pattern of melted polyethylene at $190^{\circ} \mathrm{C}$ in case of the deformation velocity of $0.0068 \mathrm{~s}^{-1}$. The flow pattern there is concave to the bottom, indicating the condition near the critical deformation velocity. The deformation velocity being $0.0077 \mathrm{~s}^{-1}$, circulating secondary flow is clearly observed, and the region of this secondary flow increases correspondingly as the deformation velocity increases.

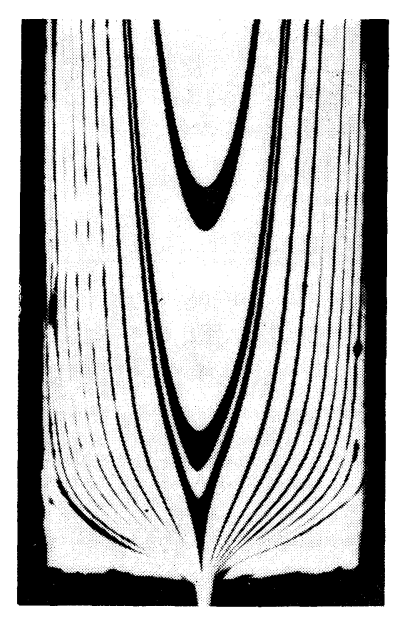

Fig. 7 Flow pattern at the incipient stage of circulating secondary flow

Table 3 The relation between circulating secondary flow and deformation velocity

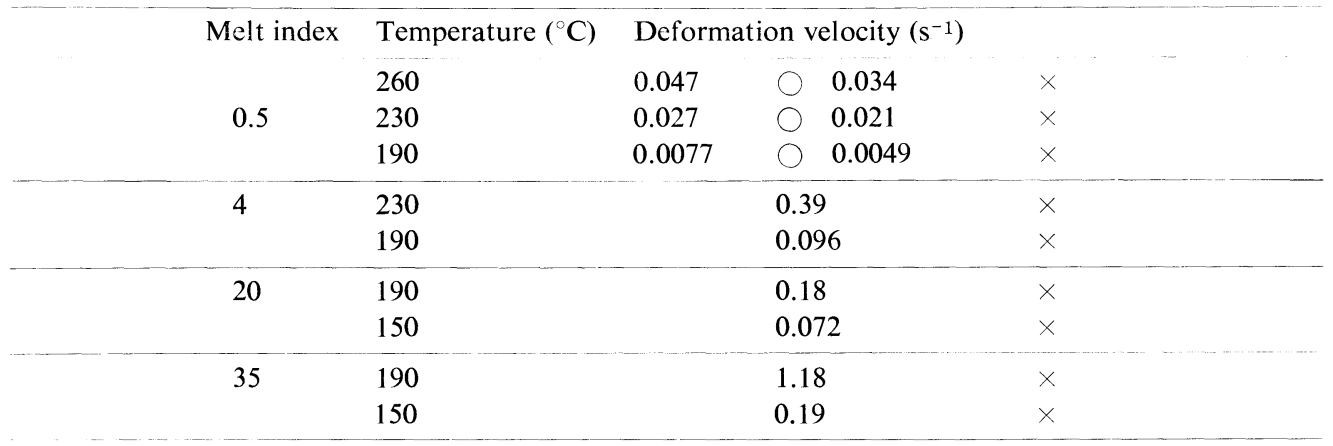

Table 4 The relation between circulating secondary flow and Reynolds numbers

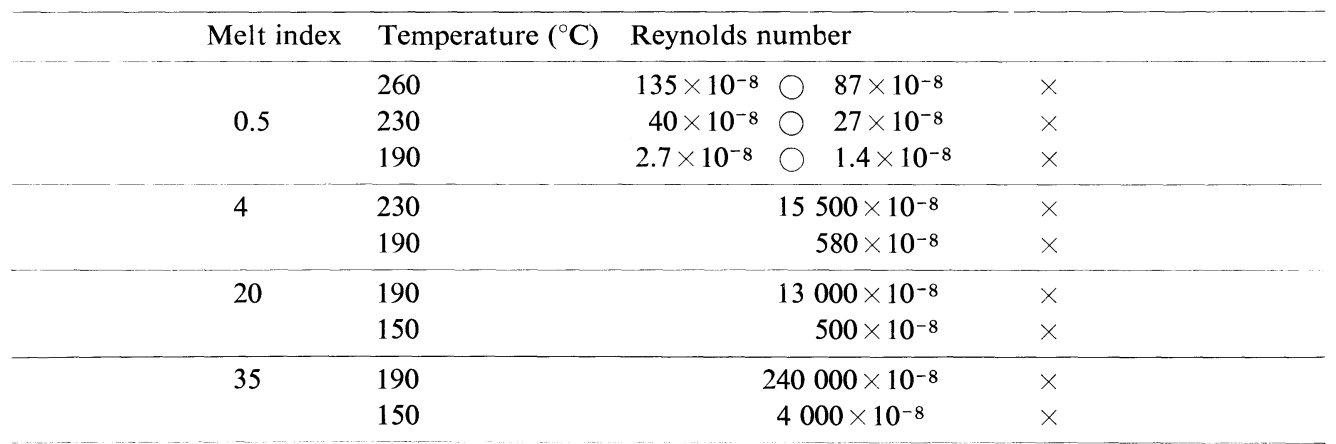




\section{b. Influence of temperature}

From the results of Table 3, the deformation velocity at which the secondary flow appears increases as the temperature of polymeric melts becomes higher. Fig. 8 in which two flow patterns at different temperatures are compared indicates that the region of circulating secondary flow is larger at low temperature than at high temperature at the same deformation velocity.

We stated previously that the critical deformation velocity is high for large melt index, that is at small relaxation time. Availing of this idea, we consider next the influence of the temperature of melted polyethylene on the critical deformation velocity. From eq. (9), the higher the temperature is the shorter the relaxation time or the less the viscoelastic quality is, resulting in that the critical deformation velocity for appearing circulating secondary flow is higher. This agrees with these experimental results.

6.2 For melt index above 4

As all flow patterns of melted polyethylene for melt
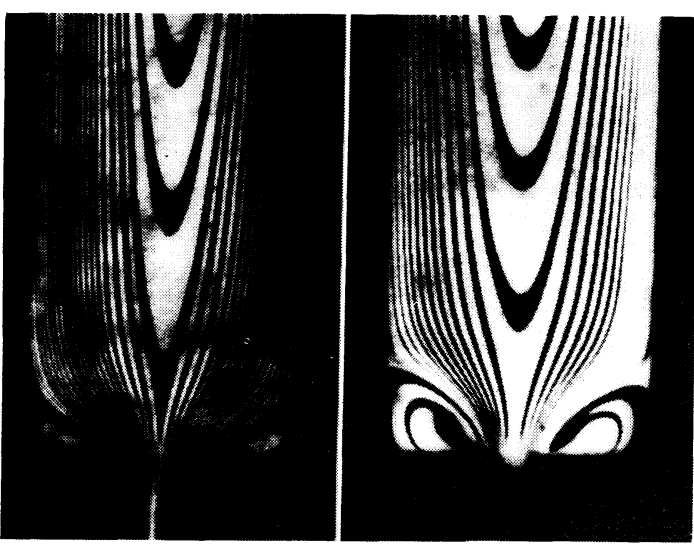

Fig. 8 Flow patterns at different temperatures (melt index 0.5, deformation velocity $0.052 \mathrm{~s}^{-1}$ ) indices of 4, 20 or 35 showed the silimar tendency within the limits of this experiment, the results are mentioned together.

The deformation velocities shown in Table 3 were the maximum values observed in this experiment, and circulating secondary flow did not exist under these values.

For melt index 4, we observed a singular flow pattern near the slit entry shown in Fig. 9 at the deformation velocity of $0.16 \mathrm{~s}^{-1}$. This flow pattern was unstable and appeared intermittently and irregularly. There were the similar tendency for melt index 20 or 35 , and when the deformation velocity becomes near to $0.18 \mathrm{~s}^{-1}$ or $1.18 \mathrm{~s}^{-1}$, flow lines changed as the time passed. For melt index more than 4 having less viscoelastic effect, an unstable flow was observed before circulating secondary flow appeared near the slit entry. On the contrary, for melt index 0.5 having large viscoelastic effect, stable circulating secondary flow was observed before an unstable flow appeared.
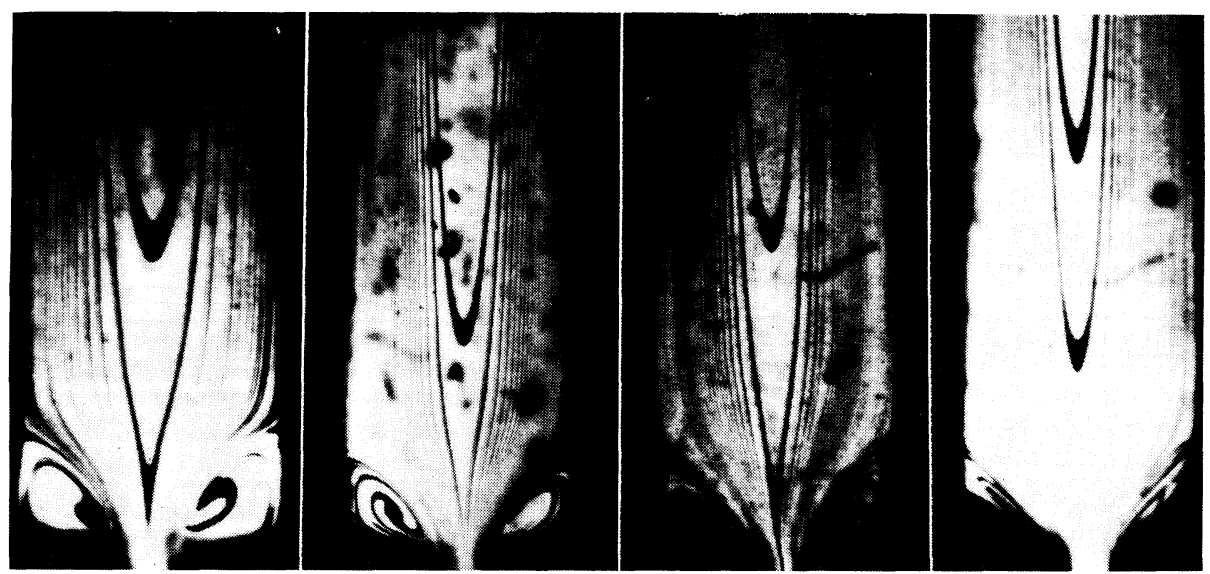

Fig. 10 Influence of entrance angle $\phi$ on flow patterns (deformation velocity $0.11 \mathrm{~s}^{-1}$, temperature $190^{\circ} \mathrm{C}$ ) 
Table 5 The relation between the entrance slit angle for appearing circulating secondary flow and the deformation velocity (at $190^{\circ} \mathrm{C}$ for melt index 0.5 )

\begin{tabular}{ccccc}
\hline Entrance angle $\phi\left({ }^{\circ}\right)$ & \multicolumn{4}{c}{ Deformation velocity $\left(\mathrm{s}^{-1}\right)$} \\
\hline 90 & 0.0077 & $\bigcirc$ & 0.0049 & $\times$ \\
80 & 0.0191 & $\bigcirc$ & 0.0167 & $\times$ \\
70 & 0.0476 & $\bigcirc$ & 0.0402 & $\times$ \\
60 & 0.0834 & $\bigcirc$ & 0.0618 & $\times$ \\
\hline
\end{tabular}

\section{Flow patterns in tapered entry}

We studied the effect of entrance angles of slit dies on flow patterns, using polyethylene for melt index 0.5 , changing deformation velocity and temperature. In Fig. 10 are shown flow patterns obtained by changing the entrance angle at the deformation velocity of $0.11 \mathrm{~s}^{-1}$ and at $190^{\circ} \mathrm{C}$. They indicate that the main flow patterns do not change, even if the entrance angles are changed. Regions of circulating secondary flow decreased with decreasing the entrance angle $\phi$.

In Table 5 is shown the relation between deformation velocities and entrance angles for appearing circulating secondary flow. It shows that the critical deformation velocity for appearing circulating secondary flow increases with decreasing the entrance angle $\phi$. This may be because the entrance angle of main flow $\phi$ (see Fig. 4) hardly changes even if the slit entrance angle $\phi$ changes, resulting in the disappearance of the circulating secondary flow when $\phi=\phi$. Hence, if the deformation velocity becomes high, the region of circulating secondary flow spreads out and the entrance angle of main flow $\phi$ becomes small, causing the entrance slit angle $\phi$ for disappearing circulating secondary flow becomes small. Thus, the critical deformation velocity for appearing circulating secondary flow becomes large with decreasing the entrance slit angle $\psi$.

\section{Conclusions}

Flow patterns of polymeric melts (low-density polyethylene having melt indexes of $0.5,4,20$ and 35) near the entrance of slit dies are observed at low Reynolds numbers, and the results are follows:

When Reynolds numbers are lower than $10^{-2}$, the circulating secondary flows appear or disappear depending upon the conditions of the main flow and of the characteristics of melted polymers.
(1) The melted polyethylene for melt index 0.5 having long relaxation time has a critical deformation velocity for appearing circulating secondary flow. The region of circulating secondary flow spreads out with increasing the deformation velocity.

(2) The melted polyethylene for melt index 4, 20 and 35 having short relaxation time indicates intermittent and irregular flow patterns near the slit, before circulating secondary flow appears.

(3) As the temperature of melted polyethylene for melt index 0.5 rises, the critical deformation velocity for appearing circulating secondary flow becomes large. This region diminishes at a constant deformation velocity.

(5) Main flow patterns hardly change even if the entrance angle is changed at a constant deformation velocity and a constant temperature.

\section{References}

[1] J. Tordella; J. Appl. Phys., 27, 454 (1956).

[2] H. Giesekus; Rheol. Acta, 7, 127 (1968).

[3] P. Schümmer; Rheol. Acta, 6, 192 (1967).

[4] T. F. Ballenger and J. L. White; J. Appl. Poly. Sci., 15, 1949 (1971).

[5] E. B. Bagley and A. M. Birks; J. Appl. Phys., 31, 556 (1960).

[6] C. D. Han; Rheol. Acta, 14, 173 (1975).

[7] L. H. Drexler and C. D. Han; J. Appl. Poly. Sci., 17, 2355 (1973).

[8] S. Middleman; The Flow of High Polymers, Interscience Publishers (1968).

[9] T. Ota et al.; Polyethylene (in Japanese), Nikkankogyo, Tokyo (1961).

[10] P. N. Kaloni; J. Phys. Soc. Japan, 20, 132 (1965).

[11] P. N. Kaloni; J. Phys. Soc. Japan, 20, 610 (1965). 